\title{
Phase-Resolved Mapping of the Near-Field Vector and Polarization State in Nanoscale Antenna Gaps
}

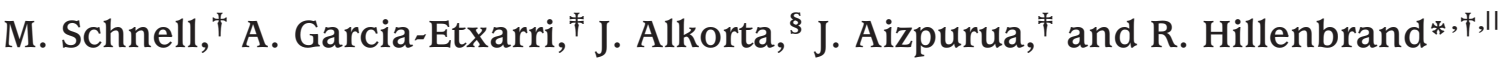 \\ ${ }^{\dagger}$ Nanooptics Group, CIC nanoGUNE Consolider, 20018 Donostia-San Sebastian, Spain, ${ }^{*}$ Centro de Fisica de \\ Materiales (CSIC-UPV/EHU) and Donostia International Physics Center (DIPC), 20018 Donostia - San Sebastian, Spain, \\ ${ }^{\S}$ CEIT and TECNUN, University of Navarra, 20018 Donostia - San Sebastian, Spain, and "IKERBASQUE, Basque \\ Foundation for Science, 48011 Bilbao, Spain
}

\begin{abstract}
We demonstrate that the local near-field vector and polarization state on planar antenna structures and in nanoscale antenna gaps can be determined by scattering-type near-field optical microscopy (s-SNOM). The near-field vector is reconstructed from the amplitude and phase images of the in- and out-of-plane near-field components obtained by polarization-resolved interferometric detection. Experiments with a mid-infrared inverse bowtie antenna yield a vectorial near-field distribution with unprecedented resolution of about $10 \mathrm{~nm}$ and in excellent agreement with numerical simulations. Furthermore, we provide first direct experimental evidence that the nanoscale confined and strongly enhanced fields at the antenna gap are linearly polarized. s-SNOM vector-field mapping paves the way to a full near-field characterization of nanophotonic structures in the broad spectral range between visible and terahertz frequencies, which is essential for future development and quality control of metamaterials, optical sensors, and waveguides.
\end{abstract}

KEYWORDS Vector near-field mapping, near-field polarization state, phase-resolved near-field microscopy, infrared antennas, bowtie aperture, local field enhancement

$\mathrm{P}$ lasmonic nanostructures and metamaterials enable the engineering of optical fields on the nanometer scale, which is the basis for the development of ultrasensitive molecular spectroscopy, high-resolution microscopy, and nanoscale optical circuits at frequencies ranging from the visible to the microwave spectral regime. ${ }^{1-4} \mathrm{~A}$ direct experimental access to the optical near-field distribution is critical for testing the design performance, exploring design alternatives, and verifying novel theoretical concepts. Because of their three-dimensional, complex-valued polarization state (vector field), however, nanoscale confined optical near fields are highly complex and thus are challenging experimental imaging techniques. ${ }^{5,6}$

Generally, the local near field at a sample surface is described by a three-dimensional vector $\mathbf{E}=\left(E_{x}, E_{y}, E_{z}\right)$, where each near-field component $E_{j}$ is characterized by both a field amplitude $\left|E_{j}\right|$ and a phase $\varphi_{j}{ }^{6,7}$ While strong field amplitudes at specific sample locations open new avenues for example in vibrational spectroscopy of single molecules, ${ }^{8-10}$ it is the phase distribution that is essential for nanoscale coherent control applications. ${ }^{11-13}$ The phase difference $\delta_{i j}=\varphi_{j}-\varphi_{i}$ between individual components is thereby a fundamental quantity as it determines the polarization state of the vector near field. ${ }^{6}$ For example, a phase difference of $\delta_{i j}=0^{\circ}$ or $180^{\circ}$ (for all $i, j$ ) defines linearly

*Corresponding author, r.hillenbrand@nanogune.eu.

Received for review: 05/12/2010

Published on Web: 08/11/2010 polarized local fields, while $\delta_{i j}= \pm 90^{\circ}$ and $\left|E_{i}\right|=\left|E_{j}\right|$ yields circularly polarized near fields. Engineering of the individual phases thus can be applied to control ${ }^{14}$ the near field polarization state for novel nanophotonic applications in, e.g., quantum optics ${ }^{15}$ or solid state physics. ${ }^{16,17}$ To this end, it is of utmost importance to develop tools that allow for ultrahigh resolution mapping of individual near-field components in both amplitude and phase.

A variety of methods such as interferometric scanning near-field optical microscopy (SNOM) ${ }^{18-24}$ scattering-type SNOM (s-SNOM), ${ }^{18,20,22,24-30}$ two-photon induced luminescence (TPL) microscopy, ${ }^{13,31,32}$ electron energy loss spectroscopy (EELS), ${ }^{33}$ and photoemission electron microscopy $(\mathrm{PEEM})^{11}$ enable the mapping of near-field distributions and thus have provided valuable insights into plasmonic antenna and waveguide structures. Each of these methods relies on an individual physical approach to probe the near fields, however, each of them is confronted with specific limitations. For example, TPL microscopy is based on an incoherent measurement principle and thus does not provide phase information. With aperture SNOM typically the transverse electric near-field components (parallel to the sample surface) are probed while scattering-type SNOM is considered to be mostly sensitive to the vertical near-field component (perpendicular to the sample surface). Indeed, several phaseand polarization-sensitive s-SNOM studies ${ }^{18,20,22}$ report amplitude and phase images of only the out-of-plane near-field component. A recent study, ${ }^{24}$ however, shows that ampli- 
(a)

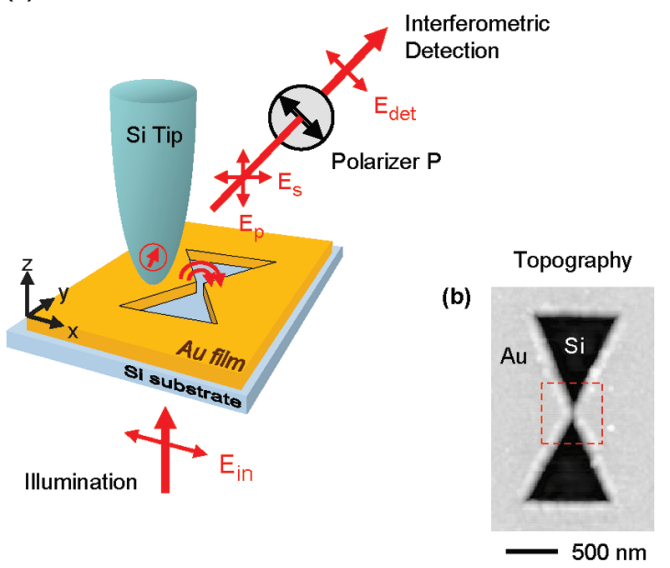

(c)

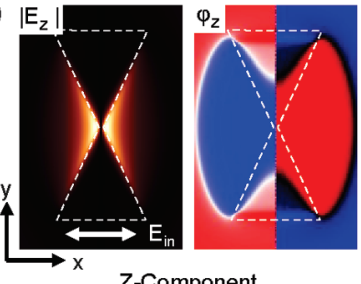

(d)

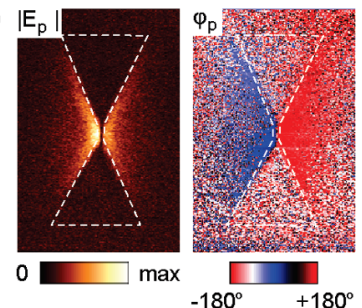

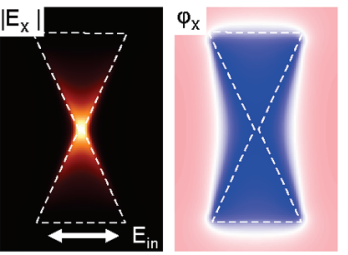

X-Component

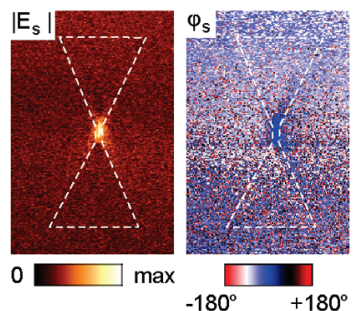

FIGURE 1. Near-field vector mapping of an inverse bowtie antenna. (a) Sketch of the experimental setup showing a silicon tip scattering the local fields of an inverse bowtie antenna. (b) Topography image of the inverse bowtie antenna (1600 $\mathrm{nm}$ length $\times 800 \mathrm{~nm}$ base $\times 40 \mathrm{~nm}$ thickness). (c) Numerically calculated near-field distribution of the inverse bowtie antenna, showing amplitude and phase of the $z-$ and the $x$-component of the electric field $30 \mathrm{~nm}$ above the Au surface at $\lambda=9.3 \mu \mathrm{m}$. (d) Experimental near-field images (raw data) of the inverse bowtie antenna recorded at $\lambda=9.3 \mu \mathrm{m}$. Vertical polarizer setting detects the p-polarized light and yields the near-field amplitude $\left|E_{\mathrm{p}}\right|$ and phase $\varphi_{\mathrm{p}}$. Likewise, a horizontal polarizer setting detects the s-polarized light and yields the near-field amplitude $\left|E_{\mathrm{s}}\right|$ and phase $\varphi_{\mathrm{s}}$. The phases $\varphi_{x}$ and $\varphi_{\mathrm{p}}$ were arbitrarily set to $0^{\circ}$ at the gap center. The phase difference between s- and p-polarization was determined by line scans across the gap where both polarizations were measured with a constant reference phase (Figure $3 b$ ).

tude and phase of both components can be measured with elongated tips, but the vector field was not reconstructed because of the unknown scattering characteristic of the tip. Vector field microscopy based on polarization-resolved intensity detection of light scattered from metal particle and aperture tips has been reported recently. ${ }^{5}$ Because of noninterferometric detection, however, essential phase information is lost with such a technique, and thus the polarization state could not be fully characterized. Here we demonstrate nanoscale resolved imaging of near-field vector components with the use of interferometric transmission-mode scattering-type near-field optical microscopy (s-SNOM) employing a dielectric tip as scattering probe. ${ }^{27}$ By analyzing the tipscattered light with a linear polarizer, we map the amplitude and phase of the in-plane (transverse) and out-of-plane (vertical) component of nanoscale confined fields at the gap of an infrared antenna. After characterizing the scattering tensor of the tip, we succeed to reconstruct for the first time the local polarization state of antenna near fields. Our results provide direct experimental evidence that the gap fields are linearly polarized.

We map the near fields of an inverse bowtie antenna (bowtie aperture), ${ }^{34,35}$ which was designed to exhibit a dipolar resonance in the mid-infrared region at a wavelength of about $9 \mu \mathrm{m}$ (Figure $1 \mathrm{a}$ ). The topography of the antenna is depicted in Figure 1b, showing the two triangular holes that were milled by focused ion beam (FIB) into a $40 \mathrm{~nm}$ thick Au film evaporated on a Si substrate. The gap of the inverse bowtie antenna has a width of approximately $80 \mathrm{~nm}$.

Distortion-free imaging of the antenna near fields (Figure 1a) was performed with our transmission-mode S-SNOM, ${ }^{20,27}$ employing the sharp dielectric tip of conventional silicon cantilevers (Nanosensors, PPP-RT-NCHR). The antenna is illuminated from below at a wavelength of $\lambda=$ $9.3 \mu \mathrm{m}$. Note that in contrast to normal bowtie antennas, the polarization of the incident field has to be chosen perpendicular to the long axis for resonant excitation (Babinet's principle ${ }^{7,36}$ ), i.e., parallel to the $x$-axis (see schematics in Figure $1 \mathrm{a}$ ). While scanning the antenna, the Si tip scatters the local near fields and the tip-scattered light is collected in the $y$-direction at an angle of $30^{\circ}$ relative to the sample surface. After passing the polarizer P (www.lasnix.com, model LP01), the tip-scattered light is detected with an interferometer. The polarization of the reference arm of the interferometer is fixed to $+45^{\circ}$ in all experiments. Suppression of background light is achieved by vertical tip oscillation (about $100 \mathrm{~nm}$ amplitude) at the cantilever's mechanical resonance frequency $\Omega=300 \mathrm{kHz}$ and demodulation of the detector signal at higher harmonics $n \Omega$. A pseudoheterodyne interferometric detection technique ${ }^{37}$ (www.neaspec.com) yields the near-field optical amplitude $|E|$ and phase $\varphi$ simultaneously. ${ }^{20,27}$

In Figure $1 \mathrm{~d}$ we demonstrate that polarization- and phaseresolved s-SNOM enables the mapping of amplitude and phase distribution of individual near-field components. We show the experimental near-field images of the inverse bowtie antenna obtained for a vertical and horizontal orientation of the polarizer, corresponding to the detection of the $\mathrm{p}$ - and s-polarized tip-scattered light, respectively. Detecting the p-component, we observe high-amplitude signals $\left|E_{\mathrm{p}}\right|$ on top of the metal film near the rim and particularly at the two edges forming the gap. Inside the gap no amplitude signal is observed but instead a phase jump of exactly $180^{\circ}$ (as already observed with IR gap antennas ${ }^{20}$ ). By rotating the polarizer to a horizontal orientation, we select the s-component of the tip-scattered light. We can immediately see that the amplitude image $\left|E_{\mathrm{s}}\right|$ exhibits a completely 
different pattern (right-hand side of Figure 1d), featuring a highly localized, intense spot inside the gap and a spatially constant phase. To analyze the near-field contrast in our images, we performed FDTD calculations (www.lumerical.com, FDTD solutions) of the fields of an inverse bowtie antenna on top of a Si substrate. Figure 1c shows the amplitude and phase of the near field components $E_{z}$ and $E_{x}$ calculated at a height of $30 \mathrm{~nm}$. The experimental images $\left(\left|E_{\mathrm{p}}\right|, \varphi_{\mathrm{p}}\right)$ and $\left(\left|E_{\mathrm{s}}\right|, \varphi_{\mathrm{s}}\right)$ are in excellent agreement with the $z$ - and $x$-components of the calculated near-field distribution, respectively. We conclude that by selecting the p-polarized scattered field (vertical polarizer setting), the signal amplitude $\left|E_{\mathrm{p}}\right|$ yields the out-of-plane field $\left|E_{z}\right|$. The signal phase $\varphi_{\mathrm{p}}$ yields $\varphi_{z}$. Analogously, we map $\left|E_{x}\right|$ and $\varphi_{x}$ by a horizontal polarizer setting (s-polarized). Remarkably, the spot size observed in the s-polarized image is about $50 \mathrm{~nm}$ (FWHM), providing direct experimental evidence that gap antennas generate in-plane fields of extreme subwavelength scale confinement $(\lambda / 200)$.

For further insights into the nature of the gap fields, we imaged the gap region at higher spatial resolution and with different polarizer angles $\beta$. The topography image of the gap region is shown in Figure 2a. The high-resolution near-field images in Figure 2c taken at the polarizer angles of $\beta=90^{\circ}$ and $0^{\circ}$ (corresponding to the detection of $\mathrm{p}$ - and s-polarizer light, respectively) clearly reproduce our results from Figure 1. Rotating the polarizer clockwise from $90^{\circ}$ toward $0^{\circ}$, interestingly, we observe asymmetric near-field patterns. While the amplitude signals at the right side of the gap become increasing extinguished, the localized spot on the left rim of the gap continuously moves toward the gap center. This observation can be understood with a sketch of our experimental configuration (Figure $2 \mathrm{~b}$ ), showing a cross section of the gap with electric field lines and the polarization-resolved near-field detection scheme. Clearly, the amplitude images taken at arbitrary polarizer angles $\beta$ highlight the antenna regions where the near fields point in the direction of $\beta$. We note that the experimental images show a much stronger field confinement at the gap center which can be explained by the fact that the tip follows the surface topography.

Our results unambiguously show the capability of s-SNOM with standard Si tips to map the vectorial nature of nanoscale antenna fields. From our experimental results in Figure 1 we can conclude that the transfer of the antenna near field $\mathrm{E}$ into the s-SNOM signal $\left(E_{\mathrm{s}}, E_{\mathrm{p}}\right)$ can be described in Cartesian coordinates by a diagonal tensor $\boldsymbol{\sigma}$

$$
\left(\begin{array}{c}
E_{\mathrm{s}} \\
E_{\mathrm{p}}
\end{array}\right) \propto\left(\begin{array}{cc}
\sigma_{\mathrm{s}} & 0 \\
0 & \sigma_{\mathrm{p}}
\end{array}\right)\left(\begin{array}{l}
\left|E_{x}\right| \mathrm{e}^{\mathrm{i} \varphi_{x}} \\
\left|E_{z}\right| \mathrm{e}^{\mathrm{i} \varphi_{z}}
\end{array}\right)
$$

The tensor $\boldsymbol{\sigma}$ essentially describes the scattering characteristics of the probing tip. $E_{\mathrm{s}}$ and $E_{\mathrm{p}}$ are the complexvalued signals that are measured when selecting the s- and p-polarized components of the tip-scattered light with the (a)
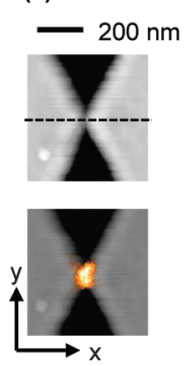

(b)

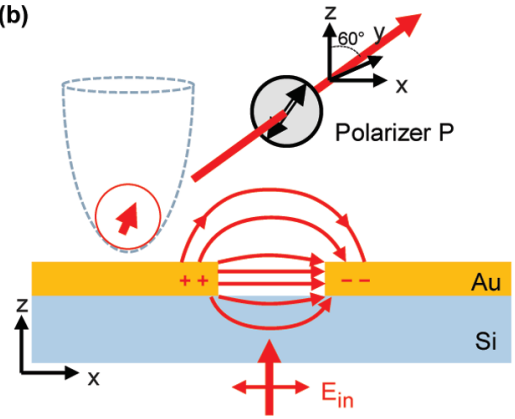

(c)
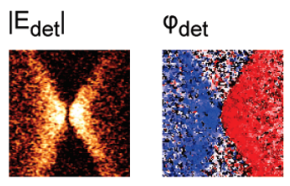

(d)
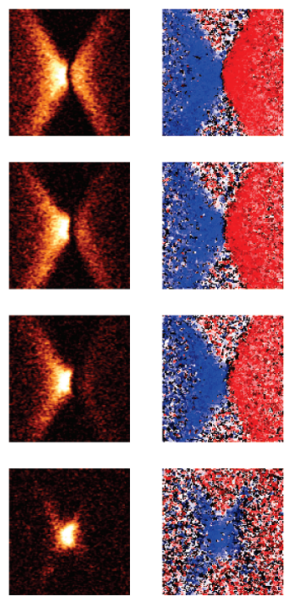

-
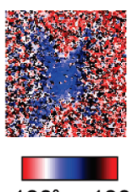

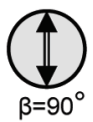

$\left|E_{\text {det }}\right|$

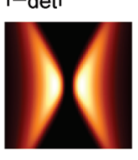

$\varphi_{\text {det }}$
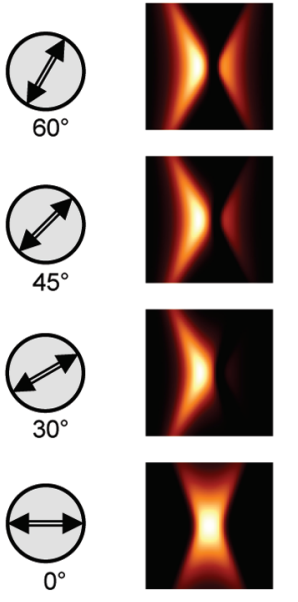

-
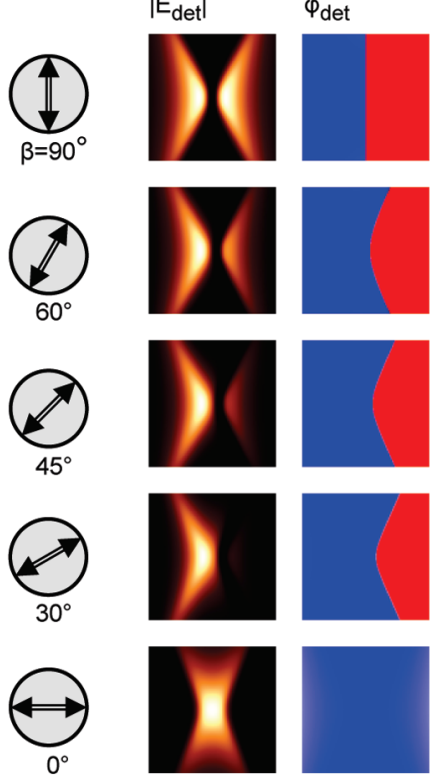

$200 \mathrm{~nm}$

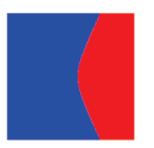

FIGURE 2. Polarization-resolved near-field study of the gap region. (a) Top: Topography image of the gap region marked by the red dashed square in Figure 1b. Bottom: Superposition of the near-field amplitude image $\left|E_{s}\right|$ on top of the topography image. (b) Illustration of the experiment, showing the cross section of the gap region. Polarization-resolved near-field detection is performed by analyzing the tip-scattered light with the polarizer P. (c) Experimental nearfield images of the gap region, recorded at different polarizer angles $\beta$ between $90^{\circ}$ and $0^{\circ}$. (d) Calculated near-field images $E_{\text {det }}$ based on eq 2 for the different polarizer angles $\beta$, using the calculated nearfield distribution of Figure $1 \mathrm{c}$ and relative tensor components $2 \sigma_{\mathrm{s}}=$ $\sigma_{\mathrm{p}}$.

use of the polarizer P. $E_{x, z}$ are the local antenna near fields in $x$ and in $z$ directions expressed by their amplitudes $\left|E_{x, z}\right|$ and phases $\varphi_{x, z}$. We note that generally the tensor $\boldsymbol{\sigma}$ connects the three-dimensional near-field vector $\left(E_{x}, E_{y}\right.$, $\left.E_{z}\right)$ with the two-dimensional far-field vector $\left(E_{\mathrm{s}}, E_{\mathrm{p}}\right)$. Due to the symmetry of the bowtie antenna, however, we can neglect the $y$ component of the electric field. Numerical calculations confirm that $E_{y} \ll E_{x, z}$. We test eq 1 by a numerical simulation of the experimental image series shown in Figure $2 \mathrm{~d}$. We calculate the recorded nearfield amplitude and phase images, $E_{\text {det }}$ and $\varphi_{\text {det }}$, according to 


$$
\left|E_{\text {det }}\right| e^{i \varphi_{\text {det }}} \propto \sigma_{\mathrm{s}}\left|E_{x}\right| e^{i \varphi_{x}} \cos \beta+\sigma_{\mathrm{p}}\left|E_{z}\right| \mathrm{e}^{i \varphi_{z}} \sin \beta
$$

where $\left|E_{x}\right|,\left|E_{z}\right|, \varphi_{x}$, and $\varphi_{z}$ are the calculated near field amplitude and phase of the $x$ and $z$ components shown in Figure $1 \mathrm{c}$. We find a good agreement between experiment and calculations when taking $2 \sigma_{\mathrm{s}}=\sigma_{\mathrm{p}}$. The result of this calculation is shown in Figure 2d. We explain the anisotropy of tensor $\boldsymbol{\sigma}$ by the elongated shape of the Si tip. Note that the tensor components $\sigma_{\mathrm{s}}$ and $\sigma_{\mathrm{p}}$ are found to be real-valued numbers. Thus, no phase shift is introduced by the tensor $\boldsymbol{\sigma}$ when the near-field components are transferred to the far-field signals $E_{\mathrm{s}}$ and $E_{\mathrm{p}}$. We explain the real-valued tensor components by the dielectric (nonresonant) material properties of the Si tip.

In the following we demonstrate that knowledge of the tensor $\boldsymbol{\sigma}$ allows for reconstruction of the vectorial near-field distribution and the polarization state of the gap fields. In Figure $3 \mathrm{~b}$ we plot amplitude and phase of the $x$ - and $z$-component of the gap fields across the gap center according to

$$
\left(\begin{array}{l}
\left|E_{x}\right| e^{i \varphi_{x}} \\
\left|E_{z}\right| e^{i \varphi_{z}}
\end{array}\right)=\frac{1}{\sigma_{\mathrm{s}}^{-1}\left|E_{\mathrm{s}}(0)\right|}\left(\begin{array}{l}
\sigma_{\mathrm{s}}^{-1}\left|E_{\mathrm{s}}\right| e^{i \varphi_{\mathrm{s}}} \\
\sigma_{\mathrm{p}}^{-1}\left|E_{\mathrm{p}}\right| \mathrm{e}^{i \varphi_{\mathrm{p}}}
\end{array}\right)
$$

with $2 \sigma_{\mathrm{s}}=\sigma_{\mathrm{p}}$. The experimental values for $\left(E_{\mathrm{s}}, \varphi_{\mathrm{s}}\right)$ and $\left(E_{\mathrm{p}}, \varphi_{\mathrm{p}}\right)$ have been extracted from line scans across the antenna gap (along the dashed line in Figure 2a) for the polarizer angles $\beta$ $=0^{\circ}$ and $\beta=+90^{\circ}$. It is important to note that the reference phase in this experiment is constant for both polarizer settings, thus yielding the phase difference between the individual polarizations. We also note that in this experiment the measured signals are not referenced to the incident field. Thus, we measure the ratio between the field components rather than the field enhancement compared to the incident field. For comparison, we show in Figure $3 \mathrm{c}$ the numerically calculated near-field components (normalized to $\left|E_{x}(0)\right|$ ) along the dashed line in Figure 3a, thus taking into account that the tip follows the surface topography. In both experiment and calculations we observe strong in-plane field amplitudes inside the gap $(x=0)$ which exceed the maximum out-of-plane fields by a factor of about 2.5. Remarkably, also the double-peak structure of the $x$ component inside the gap can be visualized with a resolution of about $10 \mathrm{~nm}$. It reveals that the infrared fields are not localized in the center of the gap but rather at the two metal edges, as also predicted by the numerical calculations in Fig. 3a. The phase measurement (right-hand side of Figure 3b) reveals a phase difference between the $x$ and $z$ components amounting to $\delta_{x z}=\varphi_{z}-\varphi_{x} \approx 180^{\circ}$ for $x>0$ and $\delta_{x z} \approx 0^{\circ}$ for $x<0$.

The polarization state of an optical field can generally be visualized by a parametric plot of the electric field vector $\vec{E}(t)$ as a function of time $t$

$$
\vec{E}(t)=R e\left(\begin{array}{l}
\left|E_{x}\right| \mathrm{e}^{i\left(-\omega t+\varphi_{x}\right)} \\
\left|E_{z}\right| \mathrm{e}^{i\left(-\omega t+\varphi_{z}\right)}
\end{array}\right)
$$

where $\omega$ is the angular light frequency. ${ }^{7}$ In Figure $3 \mathrm{~d}$ we display the polarization state of the antenna gap fields at the positions $x=-30,0$, and $+30 \mathrm{~nm}$ using the experimental and numerically calculated near-field amplitude and phase values taken from Figure 3b,c. The experimental plots (green curves) yield strongly elliptical (nearly linear) trajectories, providing direct evidence of linearly polarized near fields.
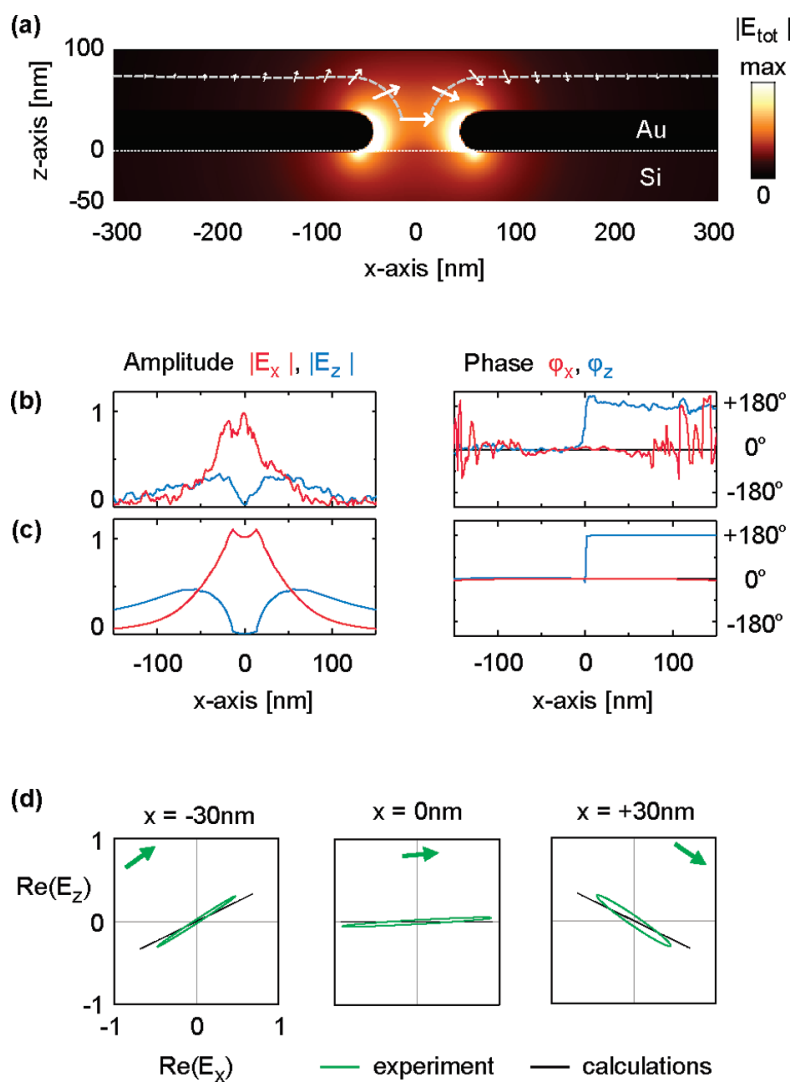

FIGURE 3. Characterization of the near-field polarization state at the antenna gap. (a) Cross section of the antenna gap showing the numerically calculated total electric field $\left|E_{\text {tot }}\right|$. The direction of the local fields (white arrows) is plotted along the height contour that the tip follows in the experiment (dashed line). (b) Experimental amplitude and phase of the in-plane $\left(\left|E_{x}\right|, \varphi_{x}\right)$ and out-of-plane $\left(\left|E_{z}\right|\right.$, $\varphi_{z}$ ) near-field components across the antenna gap reconstructed with eq 3 from experimental line scans (along the black dashed line in Figure 2a). A spatially constant phase shift (equal for both components) has been added to the phase data $\varphi_{x}$ and $\varphi_{z}$ in order to obtain absolute phase agreement with the numerical calculation. (c) Calculated amplitude and phase of the in-plane $\left(\left|E_{x}\right|, \varphi_{x}\right)$ and outof-plane $\left(\left|E_{z}\right|, \varphi_{z}\right)$ components of the near field along the dashed line shown in (a). The phase $\varphi_{x}$ was set to $0^{\circ}$ at the gap center. (d) Polarization state of the near fields at positions $x=-30,0$, and 30 $\mathrm{nm}$ obtained from eq 4 . The green line displays the polarization state obtained from the experimental near-field components shown in Figure $3 \mathrm{~b}$. The black lines display the polarization state obtained from the numerically calculated near-field components shown in Figure 3c. The green arrows indicate the instantaneous orientation of the experimental near field at time $t=0$. 
This finding is confirmed by plots of the numerically calculated electric field vector (black lines), which show a perfect linear polarization state of the gap fields. We note that the slight discrepancy between experiment and calculation is due to experimental uncertainties in the phase measurement. Furthermore, we can measure the local field direction. While inside the gap $(x=0 \mathrm{~nm})$ the fields are horizontally oriented, we find a field orientation of about $\pm 30^{\circ}$ at positions $x= \pm 30 \mathrm{~nm}$ relative to the sample surface.

We note that all three components of antenna vector fields could be measured by a rotation of the sample and of the polarization of the incident light. Because of the transmission-mode setup, the antenna illumination and thus the near-field distribution are unaffected by such rotation. This is an essential advantage compared to s-SNOM with side illumination ${ }^{18,22,23}$ where the antenna illumination is dependent on the sample orientation. However, in the case of structures with two non-negligible in-plane components, a mixture of in- and out-of-plane components will be measured with the present experimental setup (detection of the tip-scattered light at an angle of $30^{\circ}$ relative to the sample surface). Thus a more sophisticated image analysis or a modification of the present setup is required, allowing for the separation of the individual near-field components. For example, mixing of the components could be avoided by detection of the tip-scattered light at small angles relative to the sample surface.

In conclusion, we succeeded to map the in- and out-of-plane components of the vector field of an IR antenna by employing standard dielectric atomic force microscopy tips as scatteringtype near-field probes. In contrast to nanoparticle tips ${ }^{5}$-with typical diameters of about $100 \mathrm{~nm}$-we are able to access and probe the fields inside narrow antenna gaps with nanometer resolution. Because generally the phase can vary considerably across nanoantennas, ${ }^{20}$ the separated acquisition of near-field amplitude and phase is a requirement for a full and reliable characterization of the polarization state of local near fields. Phase-resolved vector-field mapping thus paves the way to a complete characterization of nanophotonic structures, analogous to a vector analyzer at radio frequencies, which will play an important role for future engineering of metamaterials, optical sensors and waveguides operating at visible, infrared, and terahertz frequencies.

Acknowledgment. Supported by the European FP7 project "Nanoantenna" (Health-F5-2009-241818) and the National Project MAT2009-08393 from the Spanish Ministerio de Ciencia e Innovacion. M.S. acknowledges financial support from "Programa de Formación de Personal Investigador" promoted by the Department of Education, Universities and Research of the Basque Government.

\section{REFERENCES AND NOTES}

(1) Engheta, N. Science 2007, 317 (5845), 1698-1702.

(2) Schuller, J. A.; Barnard, E. S.; Cai, W.; Jun, Y. C.; White, J. S.; Brongersma, M. L. Nat. Mater. 2010, 9 (3), 193-204.

(3) Shalaev, V. M. Nat. Photonics 2007, 1 (1), 41-48.
(4) Bharadwaj, P.; Deutsch, B.; Novotny, L. Adv. Opt. Photonics 2009 , 1 (3), 438-483

(5) Lee, K. G.; Kihm, H. W.; Kihm, J. E.; Choi, W. J.; Kim, H.; Ropers, C.; Park, D. J.; Yoon, Y. C.; Choi, S. B.; Woo, D. H.; Kim, J.; Lee, B.; Park, Q. H.; Lienau, C.; Kim, D. S. Nat. Photonics 2007, 1 (1), 53-56.

(6) Gersen, H.; Novotny, L.; Kuipers, L.; van Hulst, N. F. Nat. Photonics 2007, 1 (5), 242-242.

(7) Born, M.; Wolf, E. Principles of optics; Cambridge University Press: Cambridge, 1999.

(8) Nie, S.; Emory, S. R. Science 1997, 275, 1102-1106.

(9) Kneipp, K.; Wang, Y.; Kneipp, H.; Perelman, L. T.; Itzkan, I.; Dasari, R. R.; Feld, M. S. Phys. Rev. Lett. 1997, 78 (9), 1667-1670.

(10) Xu, H.; Bjerneld, E. J.; Käll, M.; Börjesson, L. Phys. Rev. Lett. 1999, 83 (21), 4357-4360.

(11) Aeschlimann, M.; Bauer, M.; Bayer, D.; Brixner, T.; Garcia de Abajo, F. J.; Pfeiffer, W.; Rohmer, M.; Spindler, C.; Steeb, F. Nature 2007, 446 (7133), 301-304.

(12) Stockmann, M. I.; Faleev, S. V.; Bergmann, D. J. Phys. Rev. Lett. 2002, 88, 67402.

(13) Volpe, G.; Cherukulappurath, S.; Juanola Parramon, R.; MolinaTerriza, G.; Quidant, R. Nano Lett. 2009, 9 (10), 3608-3611.

(14) Biagioni, P.; Huang, J. S.; Duò, L.; Finazzi, M.; Hecht, B. Phys. Rev. Lett. 2009, 102 (25), 256801.

(15) Lounis, B.; Orrit, M. Rep. Prog. Phys. 2005, 68 (5), 1129.

(16) Qiu, Z. Q.; Bader, S. D. J. Magn. Magn. Mater. 1999, 200 (1-3), 664-678.

(17) Stanciu, C. D.; Hansteen, F.; Kimel, A. V.; Kirilyuk, A.; Tsukamoto, A.; Itoh, A.; Rasing, T. Phys. Rev. Lett. 2007, 99 (4), No. 047601.

(18) Hillenbrand, R.; Keilmann, F.; Hanarp, P.; Sutherland, D. S.; Aizpurua, J. Appl. Phys. Lett. 2003, 83, 368-370.

(19) Yu, N.; Cubukcu, E.; Diehl, L.; Belkin, M. A.; Crozier, K. B.; Capasso, F.; Bour, D.; Corzine, S.; Hofler, G. Appl. Phys. Lett. 2007, $91(17), 173113$.

(20) Schnell, M.; Garcia-Etxarri, A.; Huber, A. J.; Crozier, K. B.; Aizpurua, J.; Hillenbrand, R. Nat. Photonics 2009, 3 (5), 287 291.

(21) Burresi, M.; van Oosten, D.; Kampfrath, T.; Schoenmaker, H.; Heideman, R.; Leinse, A.; Kuipers, L. Science 2009, 326 (5952), $550-553$

(22) Esteban, R.; Vogelgesang, R.; Dorfmüller, J.; Dmitriev, A.; Rockstuhl, C.; Etrich, C.; Kern, K. Nano Lett. 2008, 8 (10), 31553159.

(23) Olmon, R. L.; Krenz, P. M.; Jones, A. C.; Boreman, G. D.; Raschke, M. B. Opt. Express 2008, 16 (25), 20295-20305.

(24) Kim, Z. H.; Leone, S. R. Opt. Express 2008, 16, 1733-1741.

(25) Hillenbrand, R.; Keilmann, F. Appl. Phys. B: Lasers Opt. 2001, 73, 239-243

(26) Dorfmüller, J.; Vogelgesang, R.; Weitz, R. T.; Rockstuhl, C.; Etrich, C.; Pertsch, T.; Lederer, F.; Kern, K. Nano Lett. 2009, 9 (6), 23722377.

(27) Schnell, M.; Garcia-Etxarri, A.; Huber, A. J.; Crozier, K. B.; Borisov, A. G.; Aizpurua, J.; Hillenbrand, R. J. Phys. Chem. C 2010, 114 (16), $7341-7345$.

(28) Kim, D.-S.; Heo, J.; Ahn, S.-H.; Han, S. W.; Yun, W. S.; Kim, Z. H. Nano Lett. 2009, 9 (10), 3619-3625.

(29) Kim, D. S.; Kim, Z. H. J. Korean Phys. Soc. 2007, 52 (1), 17-20.

(30) Rang, M.; Jones, A. C.; Zhou, F.; Li, Z.-Y.; Wiley, B. J.; Xia, Y.; Raschke, M. B. Nano Lett. 2008, 8 (10), 3357-3363.

(31) Mühlschlegel, P.; Eisler, H.-J.; Martin, O. J. F.; Hecht, B.; Pohl, D. W. Science 2005, 308, 1607-1609.

(32) Ghenuche, P.; Cherukulappurath, S.; Taminiau, T. H.; Van Hulst, N. F.; Quidant, R. Phys. Rev. Lett. 2008, 101 (11), 116805.

(33) Nelayah, J.; Kociak, M.; Stephan, O.; Garcia de Abajo, F. J.; Tence, M.; Henrard, L.; Taverna, D.; Pastoriza-Santos, I.; Liz-Marzan, L. M.; Colliex, C. Nat. Phys. 2007, 3 (5), 348-353.

(34) Guo, H.; Meyrath, T. P.; Zentgraf, T.; Liu, N.; Fu, L.; Schweizer, H.; Giessen, H. Opt. Express 2008, 16 (11), 7756-7766.

(35) Wang, L.; Uppuluri, S. M.; Jin, E. X.; Xu, X. F. Nano Lett. 2006, 6 (3), 361-364.

(36) Zentgraf, T.; Meyrath, T. P.; Seidel, A.; Kaiser, S.; Giessen, H.; Rockstuhl, C.; Lederer, F. Phys. Rev. B 2007, 76 (3), No. 033407.

(37) Ocelic, N.; Huber, A.; Hillenbrand, R. Appl. Phys. Lett. 2006, 89 (10), 101124 